\title{
Dipalmitoleoylphosphoethanolamine as a PP2A Enhancer Obstructs Insulin Signaling by Promoting Ser/Thr Dephosphorylation of Akt
}

\author{
Ayako Tsuchiya Takeshi Kanno Tomoyuki Nishizaki \\ Division of Bioinformation, Department of Physiology, Hyogo College of Medicine, Nishinomiya, Japan
}

\section{Key Words}

Phosphatidylethanolamine • Protein phosphatase 2A • Akt • GLUT4 • Glucose uptake

\begin{abstract}
Background/Aims: The phospholipid phosphatidylethanolamine is implicated in the regulation of a variety of cellular processes. The present study investigated the effect of phosphatidylethanolamines such as 1,2-diarachidonoyl-sn-glycero-3-phosphoethanolamine (DAPE), 1,2-dilinoleoyl-sn-glycero-3-phosphoethanolamine (DLPE), 1,2-dioleoyl-sn-glycero3-phosphoethanolamine (DOPE), and 1,2-dipalmitoleoyl-sn-glycero-3-phosphoethanolamine (DPPE) on protein phosphatases, Akt1/2 activity, GLUT4 mobilizations, and glucose uptake into cells. Methods: Activity of protein phosphatase 2A (PP2A) was assayed under the cellfree conditions, and Western blotting, intracellular GLUT4 trafficking, and glucose uptake into cells were monitored using differentiated 3T3-L1-GLUT4myc adipocytes. Results: Of the investigated phosphatidylethanolamines, DLPE and DPPE significantly enhanced PP2A activity. DPPE inhibited insulin-induced phosphorylation of Akt1/2 at Thr308/309 and Ser473/474 in differentiated 3T3-L1-GLUT4myc adipocytes. DPPE also inhibited insulin-stimulated GLUT4 translocation to the cell surface and reduced insulin-stimulated glucose uptake into adipocytes. Conclusion: The results of the present study indicate that the PP2A enhancer DPPE obstructs insulin signaling by promoting serine/threonine dephosphorylation of $A k t 1 / 2$, resulting in the suppression of GLUT4 translocation to the cell surface and glucose uptake into adipocytes.
\end{abstract}

Copyright $\odot 2014$ S. Karger AG, Basel

\section{Introduction}

Emerging evidence has pointed to lipids as a regulator of insulin signaling. Excess free fatty acids (FFAs) in skeletal muscle cells, liver cells, and adipocytes may attenuate insulin signaling by inhibiting insulin-induced Akt activation or upregulating protein tyrosine 
phosphatase 1B (PTP1B), a negative regulator of insulin signaling [1]. Saturated FFAs such as palmitic and stearic acid upregulate expression of the PTP1B mRNA and enhance PTP1B activity $[1,2]$. Palmitic acid also reduces insulin-induced phosphorylation of Akt2 at Thr309 and Ser474 in L6 skeletal muscle cells, without affecting expression or activity of PI3K, and suppresses insulin-stimulated glucose uptake in C2C12 myotubes [2, 3]. Taken together, these results suggest that saturated FFAs could be a factor responsible for insulin resistance related to type 2 diabetes. Little, however, is known about the effect of phospholipids such as phosphatidylcholine, phosphatidylserine, phosphatidylinositol, and phosphatidylethanolamine on insulin signaling.

The present study investigated the effects of phosphatidylethanolamine on activities of protein phosphatases and insulin signaling cascades, intracellular GLUT4 mobilizations, and glucose uptake into cells. We show here that 1,2-dipalmitoleoyl-sn-glycero-3phosphoethanolamine (DPPE) serves as an enhancer of protein phosphatase 2A (PP2A), to inhibit insulin-induced Akt activation and the ensuing GLUT4 translocation to the cell surface and glucose uptake into 3T3-L1-GLUT4myc adipocytes.

\section{Materials and Methods}

Assay of protein phosphatase activities under the cell-free conditions

Activities of protein phosphatases protein phosphatase 1 (PP1), PP2A, and PTP1B under the cellfree conditions were assayed by the method as previously described [4]. The human recombinant PP1 was purchased from New England BioLabs Inc. (Ipswich, MA, USA) and the human recombinant PP2A from Wako Pure Chemical Industries, Ltd. (Osaka, Japan). The human PTP1B was cloned into pGEX-6P-3 vector with a GST tag at the $\mathrm{NH}_{2}$ terminus, and expressed in competent E. coli BL21 (DE3), suitable for transformation and protein expression. GST-fusion PTP1B was affinity-purified using Glutathione Sepharose 4B (GE Healthcare; Piscataway, NJ, USA). Each phosphatase activity was assayed by reacting with $p$-nitrophenyl phosphate (pNPP)(Sigma-Aldrich; St. Louis, MO, USA) as a substrate. PP1 (1 U/well), PP2A (0.2 U/well), or PTP1B (1 $\mu \mathrm{g} /$ well) was preincubated at $30^{\circ} \mathrm{C}$ (for PP1) or $37^{\circ} \mathrm{C}$ (for PP2A and PTP1B) for $30 \mathrm{~min}$ in a reaction medium [50 mM HEPES, $100 \mathrm{mM} \mathrm{NaCl}, 2 \mathrm{mM}$ dithiothreitol, 0.01\% (v/v) Brij-35, 1 mM MnCl${ }_{2}$, pH 7.5 for PP1; $50 \mathrm{mM}$ Tris-HCl, $0.1 \mathrm{mM}$ EGTA, 0.1\% (v/v) 2-mercaptoethanol, pH 7.0 for PP2A; and $50 \mathrm{mM}$ HEPES, 1 mM EDTA, $50 \mathrm{mM} \mathrm{NaCl}, 1 \mathrm{mM}$ dithiothreitol, pH 7.2 for PTP1B] in the presence and absence of phosphatase inhibitors or phospholipids such as 1,2-diarachidonoyl-sn-glycero-3-phosphoethanolamine (DAPE), 1,2-dilinoleoylsn-glycero-3-phosphoethanolamine (DLPE), 1,2-dioleoyl-sn-glycero-3-phosphoethanolamine (DOPE), and DPPE. Then, pNPP at a concentration of $5 \mathrm{mM}$ for PP1, $0.5 \mathrm{mM}$ for PP2A, and $10 \mathrm{mM}$ for PTP1B was added to the reaction medium followed by 60 -min incubation, and the reaction was terminated by adding $0.1 \mathrm{~N}$ $\mathrm{NaOH}$. Dephosphorylated pNPP was quantified at an absorbance of $405 \mathrm{~nm}$ with a SpectraMax PLUS384 (Molecular Devices; Sunnyvale, CA, USA).

\section{Cell culture}

3T3-L1-GLUT4myc fibroblast cell line, expressing GLUT4myc that is constructed by inserting a human c-MYC epitope (14 amino acids) into the first ectodomain of GLUT4. Cells were cultured in Dulbecco's modified Eagle's medium (DMEM) supplemented with $10 \%(\mathrm{v} / \mathrm{v})$ calf serum, penicillin (final concentration, $100 \mathrm{U} / \mathrm{ml}$ ), and streptomycin (final concentration, $0.1 \mathrm{mg} / \mathrm{ml}$ ), in a humidified atmosphere of $5 \% \mathrm{CO}_{2}$ and $95 \%$ air at $37{ }^{\circ} \mathrm{C}$. When cells had reached confluence (day 0 ), medium was changed to DMEM supplemented with $10 \%(\mathrm{v} / \mathrm{v}$ ) fetal bovine serum (FBS), $1 \mu \mathrm{M}$ dexamethasone, $0.5 \mathrm{mM} 3$-isobutyl-methyl-xanthine and 0.1 $\mathrm{mg} / \mathrm{ml}$ insulin to differentiate from fibroblast to adipocytes (3T3-L1-GLUT4myc adipocytes). At day 3, day 7 and day 11, the medium was changed to DMEM supplemented with $10 \%$ (v/v) FBS. At 14 day, cells were used for experiments. 3T3-L1-GLUT4myc adipocytes were used for all the cell experiments in the present study.

Monitoring of GLUT4 mobilizations

3T3-L1-GLUT4myc adipocytes were incubated in Krebs-Ringer-HEPES buffer (136 mM NaCl, $4.7 \mathrm{mM}$ $\mathrm{KCl}, 1.25 \mathrm{mM} \mathrm{CaCl}_{2}, 1.25 \mathrm{mM} \mathrm{MgSO}_{4}$ and $20 \mathrm{mM}$ HEPES, pH 7.5) containing $0.2 \%$ (w/v) bovine serum 
Tsuchiya/Kanno/Nishizaki: Insulin Signaling Inhibition by a PP2A Enhancer

albumin (BSA) supplemented with $10 \mathrm{mM}$ glucose for $1 \mathrm{~h}$ at $37^{\circ} \mathrm{C}$. Cells were treated with insulin in the presence and absence of DAPE or DPPE for $20 \mathrm{~min}$. Then, cells were homogenized by sonication in an ice-cold mitochondrial buffer [ $210 \mathrm{mM}$ mannitol, $70 \mathrm{mM}$ sucrose, and $1 \mathrm{mM}$ EDTA, $10 \mathrm{mM}$ HEPES, $\mathrm{pH}$ 7.5] containing $1 \%(\mathrm{v} / \mathrm{v}$ ) protease inhibitor cocktail (Nacalai Tesque, Kyoto, Japan) and subsequently, homogenates were centrifuged at $800 \mathrm{~g}$ for $5 \mathrm{~min}$ at $4{ }^{\circ} \mathrm{C}$. The supernatants were centrifuged at $11,000 \mathrm{~g}$ for $15 \mathrm{~min}$ at $4{ }^{\circ} \mathrm{C}$ and further, the collected supernatants were ultracentrifuged at $100,000 \mathrm{~g}$ for $60 \mathrm{~min}$ at $4{ }^{\circ} \mathrm{C}$ to separate the cytosolic and plasma membrane fraction. The supernatants and pellets were used as the cytosolic and plasma membrane fractions, respectively. Whether the cytosolic and plasma membrane components were successfully separated was confirmed in the Western blot analysis using antibodies against the cytosolic marker lactate dehydrogenase (LDH) and the plasma membrane marker cadherin.

Protein concentrations for each fraction were determined using a BCA protein assay kit (Thermo Fisher Scientific, Waltham, MA, USA). Plasma membrane fraction proteins were resuspended in the mitochondrial buffer containing 1\% (w/v) sodium dodecyl sulfate (SDS). Proteins for each fraction were separated by SDS-polyacrylamide gel electrophoresis (SDS-PAGE) and transferred to polyvinylidene difluoride (PVDF) membranes. After blocking with TBS-T [ $150 \mathrm{mM} \mathrm{NaCl}, 0.1 \%$ (v/v) Tween-20, and $20 \mathrm{mM}$ Tris, pH 7.5] containing 5\% (w/v) BSA, blotting membranes were reacted with an anti-c-myc antibody (Merck Millipore, Darmstadt, Germany) followed by a horseradish peroxidase (HRP)-conjugated goat antimouse IgG antibody. Immunoreactivity was detected with an ECL kit (Invitrogen, Carlsbad, CA, USA) and visualized using a chemiluminescence detection system (GE Healthcare, Piscataway, NJ, USA). Signal density was measured with an ImageQuant software (GE Healthcare).

Monitoring of phosphorylation of insulin receptor, insulin receptor substrate 1 (IRS-1), Akt1/2, mammalian target of rapamycin (MTOR), and ERK1/2

3T3-L1-GLUT4myc adipocytes were treated with insulin in the presence and absence of DPPE, and then lysed in a lysate solution (TBS-T) [ $150 \mathrm{mM} \mathrm{NaCl}, 20 \mathrm{mM}$ Tris, $0.1 \%(\mathrm{v} / \mathrm{v}$ ) Tween-20 and $0.1 \%(\mathrm{w} / \mathrm{v}$ ) SDS, pH 7.5] containing $1 \%(\mathrm{v} / \mathrm{v})$ protease inhibitor cocktail and $1 \%(\mathrm{v} / \mathrm{v})$ phosphatase inhibitor cocktail. The lysates were centrifuged at $800 \mathrm{~g}$ for $5 \mathrm{~min}$ at $4{ }^{\circ} \mathrm{C}$. Proteins were separated by SDS-PAGE and then transferred to PVDF membranes. Blotting membranes were blocked with TBS-T containing $5 \%$ (w/v) BSA and subsequently reacted with antibodies against phospho-Tyr1185-insulin receptor $\beta$ subunit (pY1185) (Bioss, Inc., Woburn, MA, USA), insulin receptor $\beta$-subunit (Thermo Fisher Scientific), phospho-Tyr1222IRS-1 (pY1222)(Cell Signaling Technology, Inc., Danvers, MA, USA), IRS-1 (Santa Cruz Biotechnology, Inc., Dallas, Texas, USA), phospho-Thr308/309-Akt1/2 (pT308/309)(Cell Signaling Technology), phosphoSer473/474-Akt1/2 (pS473/474)(Cell Signaling Technology), Akt1/2 (Cell Signaling Technology), phospho-Ser2448-mTOR (pS2448)(Cell Signaling Technology), mTOR (Cell Signaling Technology), phospho-Thr185/202Tyr187/204-ERK1/2 (pERK1/2)(Santa Cruz Biotechnology), and ERK1/2 (Santa Cruz Biotechnology). After washing, membranes were reacted with an HRP-conjugated goat anti-mouse IgG or goat anti-rabbit IgG antibody. Immunoreactivity was detected with an ECL kit (Invitrogen) and visualized using a chemiluminescence detection system (GE healthcare). Protein concentrations for each sample were determined with a BCA protein assay kit (Thermo Fisher Scientific).

Glucose uptake assay

3T3-L1-GLUT4myc adipocytes were incubated in the Krebs-Ringer-HEPES buffer containing $0.2 \%$ (w/v) BSA supplemented with $10 \mathrm{mM}$ glucose for $1 \mathrm{~h}$ at $37^{\circ} \mathrm{C}$. Then, cells were untreated and treated with insulin $(100 \mathrm{nM})$ in phosphate buffered saline (PBS) supplemented with $10 \mathrm{mM}$ glucose for $2 \mathrm{~h}$ at $37^{\circ} \mathrm{C}$. After treatment, extracellular solution was collected and glucose was labeled with $p$-aminobenzoic ethyl ester (ABEE). Then, $5 \mu \mathrm{l}$ of ABEE-labeled solution was injected onto the column (150 X $4.6 \mathrm{~mm}$ ) equipped in the high performance liquid chromatography (HPLC) system. ABEE-labeled glucose was detected at an excitation wavelength of $305 \mathrm{~nm}$ and an emission wavelength of $360 \mathrm{~nm}$ using a fluorescence detector. Glucose concentration taken up into cells was calculated by subtracting extracellular glucose concentration after incubation for $2 \mathrm{~h}$ from initial extracellular glucose concentration (10 $\mathrm{mM})$.

Statistical analysis

Statistical analysis was carried out using analysis of variance (ANOVA) followed by a Bonferonni correction and Dunnett's test. We regarded $P<0.05$ as significant difference. 


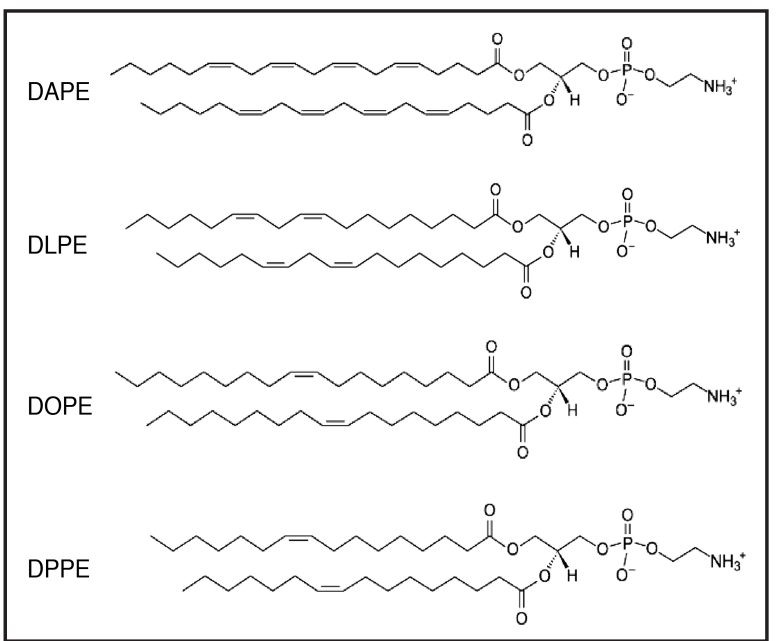

Fig. 1. Chemical structure for DAPE, DLPE, DOPE, and DPPE.

Fig. 2. Effects of phosphatidylethanolamines on activities of PP1, PP2A, and PTP1B. PP1 (A), PP2A (B), or PTP1B (C) was reacted with pNPP in the presence and absence of DAPE, DLPE, DOPE, and DPPE at a concentration of $100 \mu \mathrm{M}$ together with and without calyculin A (Caly A) (20 nM),okadaicacid(OkaA) $(2 \mathrm{nM})$,orsodiumorthovanadate $\left(\mathrm{Na}_{3} \mathrm{VO}_{4}\right)(1 \mu \mathrm{M})$, and dephosphorylated pNPP was quantified. In the graphs, each value represents the mean $( \pm$ SEM) percentage of basal phosphatase activity (Control)( $n=4$ independent experiments). $P$ values as compared with control, Dunnett's test.

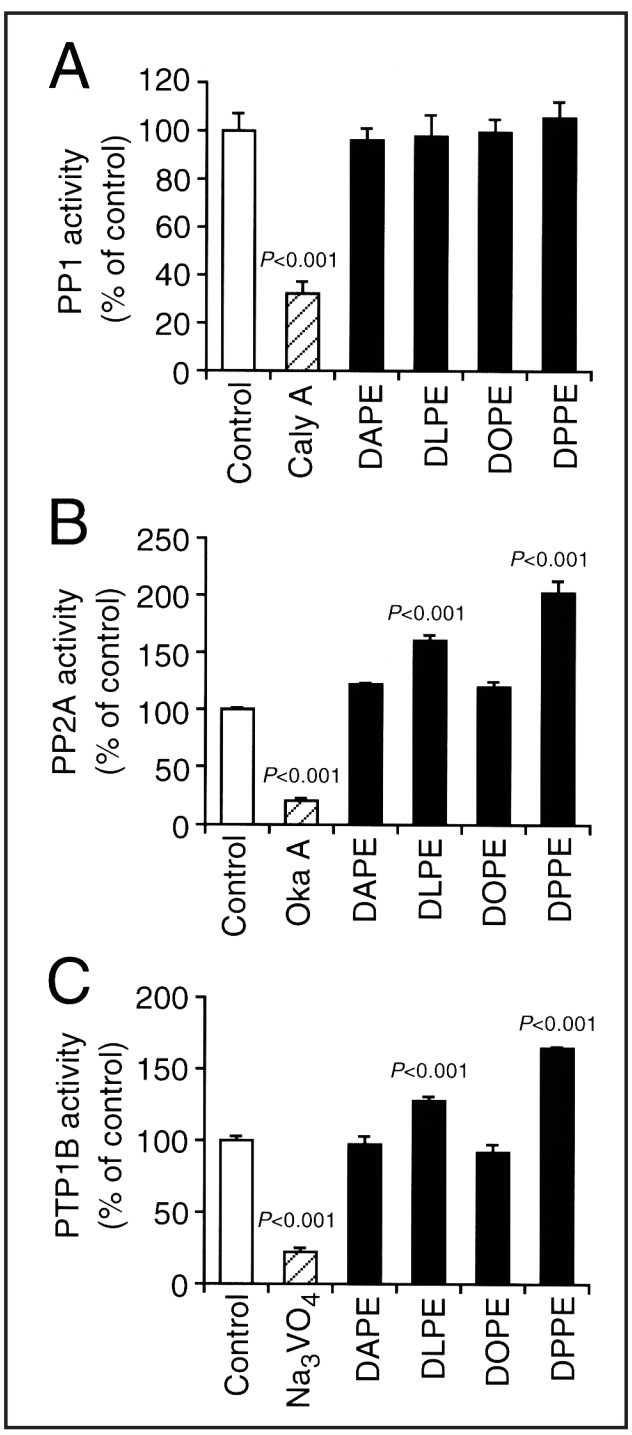

\section{Results}

DPPE enhances PP2A and PTP1B activities

We initially examined the effects of DAPE, DLPE, DOPE, and DPPE at a concentration of $100 \mu \mathrm{M}$ on protein phosphatases (Fig. 2A-C).

In the PP1 assay, calyculin A (20 nM), an inhibitor of PP1, clearly reduced PP1 activity (Fig. 2A), confirming a reliable PP1 assay. No significant effect on PP1 activity was obtained with all the investigated phosphatidylethanolamines (Fig. 2A).

In the PP2Aassay, okadaic acid ( $2 \mathrm{nM})$, an inhibitor of PP2A, clearly decreased PP2Aactivity (Fig. 2B), confirming a reliable PP2A assay. Of the investigated phosphatidylethanolamines DLPE and DPPE significantly enhanced PP2A activity, reaching 160 and 200\% of control levels, respectively, while no significant effect was obtained with DAPE and DOPE (Fig. 2B).

In the PTP1B assay, sodium orthovanadate $\left(\mathrm{Na}_{3} \mathrm{VO}_{4}\right)(1 \mu \mathrm{M})$, an inhibitor of PTP1B, clearly attenuated PTP1B activity (Fig. 2C), confirming a reliable PTP1B assay. As is the case with the effect on PP2A, DLPE and DPPE significantly enhanced PTP1B activity, reaching 130 and $160 \%$ of control levels, respectively (Fig. 2C). In contrast, DAPE and DOPE had no effect on PTP1B activity (Fig. 2C). 

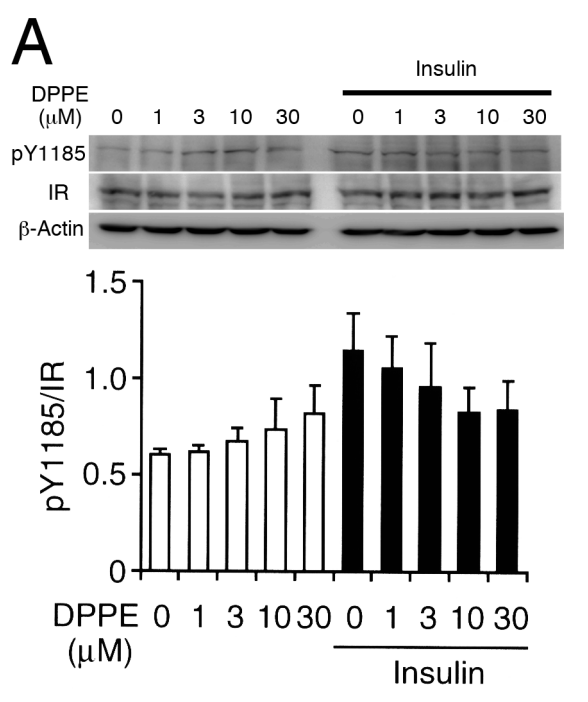

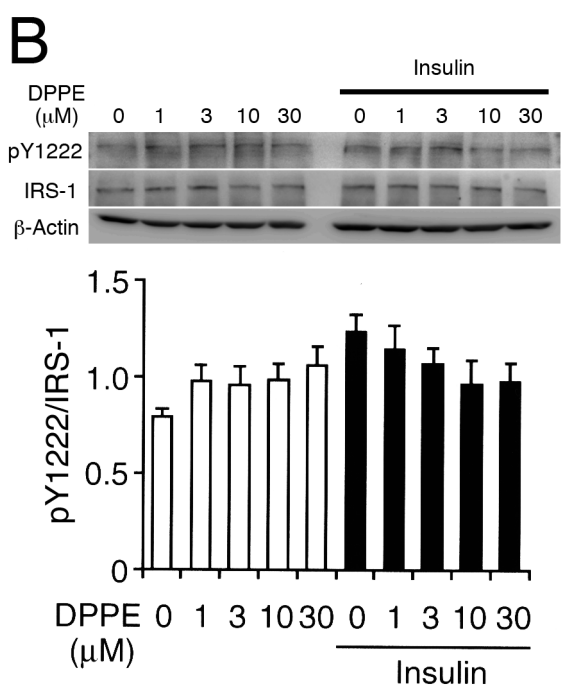

Insulin

Fig. 3. Effects of DPPE on insulin-induced phosphorylation of insulin receptor (IR) and IRS-1 in 3T3-L1GLUT4myc adipocytes. Cells were untreated and treated with insulin (Ins)(100 nM) in the presence and absence of DPPE at concentrations as indicated for $10 \mathrm{~min}$, followed by Western blotting using antibodies against pY1185, IR, pY1222, and IRS-1. Signal intensities for phosphorylation of IR and IRS-1 were normalized by those for total each protein. In the graphs, each column represents the mean $( \pm$ SEM) normalized intensity for phosphorylation of IR (A) and IRS-1 (B)(n=4 independent experiments).

\section{DPPE inhibits insulin-induced Akt1/2 activation}

Oftheinvestigated phosphatidylethanolamines DPPEhad thehighest potential to enhance PP2A and PTP1B activities. Insulin initiates insulin signals through tyrosine phosphorylation of insulin receptor and IRS-1. PTP1B dephosphorylates tyrosine phosphorylation. Then, we supposed that the PTP1B enhancer DPPE might facilitate dephosphorylation of insulininduced tyrosine phosphorylation of insulin receptor and IRS-1. To address this issue, we examined the effect of DPPE on insulin-induced tyrosine phosphorylation of insulin receptor and IRS-1 in 3T3-L1-GLUT4myc adipocytes. DPPE did not affect expression of insulin receptor, IRS-1, and $\beta$-actin in the presence and absence of insulin (Fig. 3A,B). DPPE actually attenuated insulin (100 nM)-induced phosphorylation of insulin receptor at Tyr1185 and IRS-1 at Tyr1222 in a concentration (1-30 $\mu \mathrm{M})$-dependent manner, but the effect was not significant (Fig. 3A,B). This suggests that DPPE does not enhance PTP1B activity sufficiently to dephosphorylate insulin-stimulated tyrosine phosphorylation of insulin receptor and IRS-1.

Insulin activates Akt1/2 by phosphorylating at Thr308/309 and Ser473/474 through a pathway along an insulin receptor/IRS-1/phosphatidylinositol 3 kinase (PI3K)/3phosphoinositide-dependent protein kinase-1 (PDK1) axis. PP2A dephosphorylates serine/ threonine phosphorylation, and inactivates Akt1/2 by dephosphorylating phosphorylation at Thr308/309 and Ser473/474, preferentially Thr308/309 [5]. We subsequently examined the effect of DPPE on insulin-induced serine/threonine phosphorylation of Akt1/2. DPPE significantly inhibited insulin (100 nM)-induced phosphorylation of Akt1/2 at Thr308/309 and Ser473/474 in a concentration (1-30 $\mu \mathrm{M})$-dependent manner, without affecting each basal phosphorylation in the absence of insulin (Fig. 4A). This suggests that the PP2A enhancer DPPE inhibits insulin-induced Akt1/2 activation by promoting serine/threonine dephosphorylation of Akt1/2.

Insulin, alternatively, activates mTOR by stimulating Akt-mediated phosphorylation of mTOR at Ser2448 [6-9]. Conversely, activated mTOR phosphorylates Akt1/2 at Ser473/474, leading to further enhancement of Akt1/2 activity $[10,11]$. In the present study, insulin $(100 \mathrm{nM})$ enhanced phosphorylation of mTOR at Ser2448, and the effect was significantly 
Fig. 4. Effects of DPPE on insulininduced phosphorylation of Akt1/2, mTOR, and ERK1/2. Cells were untreated and treated with insulin (Ins) $(100 \mathrm{nM})$ in the presence and absence of DPPE at concentrations as indicated for assay of Akt $1 / 2$ phosphorylation (A) and at $30 \mu \mathrm{M}$ for assay of mTOR (B) and ERK1/2 phosphorylation (C) for $10 \mathrm{~min}$, followed by Western blotting using antibodies against pT308/309, p S $473 / 474$, Akt1/2, pS2448, mTOR, pERK1/2, and ERK1/2. Signal intensities for phosphorylation of Akt1/2, mTOR, and ERK1/2 were normalized by those for total each protein. In the graphs, each column represents the mean $( \pm$ SEM) normalized intensi-

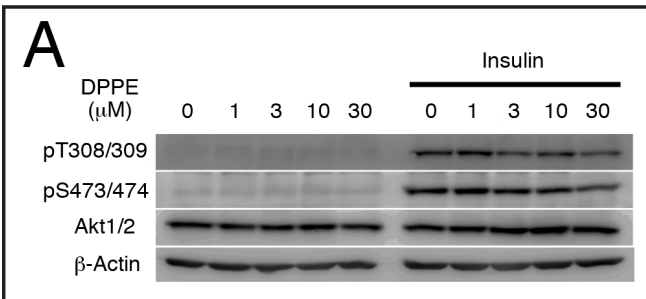

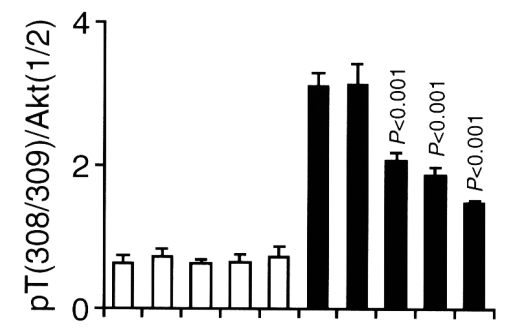

DPPE 01310300131030 $(\mu \mathrm{M})$

B
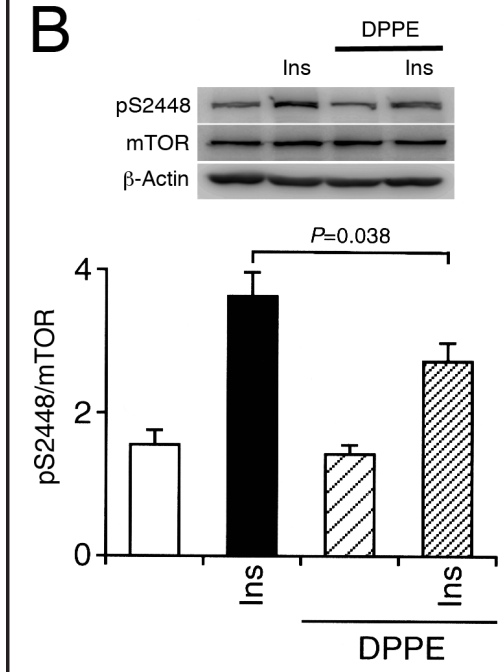

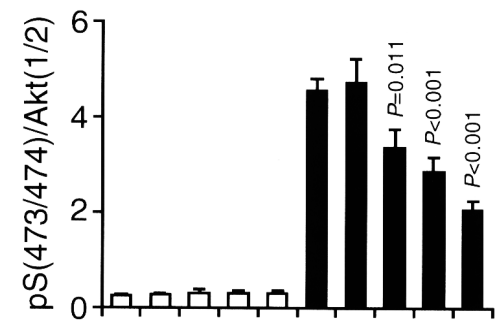

DPPE 01310300131030 $(\mu \mathrm{M})$ Insulin
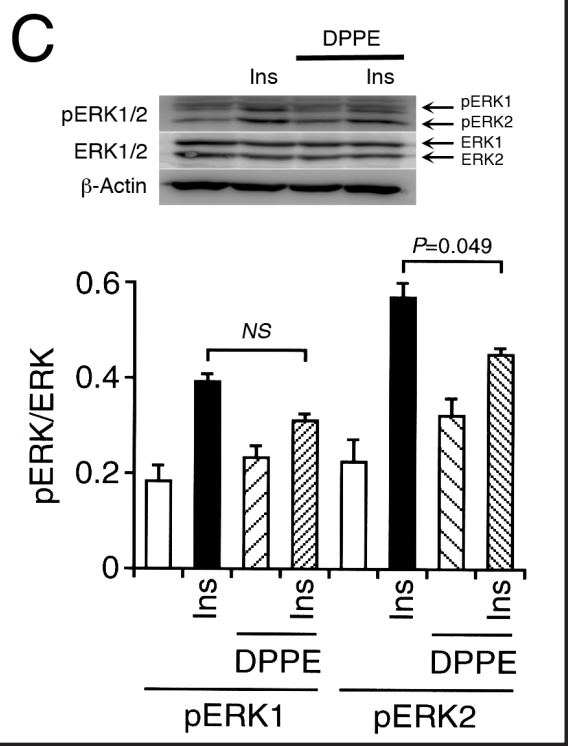

ty for phosphorylation of Akt1/2 (A), mTOR (B) and ERK1/2 (C) (n=4 independent experiments). $P$ values as compared with the intensity for cells treated with insulin in the absence of DPPE, Dunnett's test. NS, not significant.

suppressed by DPPE (30 $\mu \mathrm{M})$ (Fig. 4B). This suggests that DPPE inhibits insulin-induced activation of Akt1/2 followed by mTOR, allowing additional Akt1/2 inhibition.

We further monitored the effect of DPPE on threonine phosphorylation of ERK1/2 in another insulin signaling pathway distinct from an insulin receptor/IRS-1/PI3K/PDK1/ Akt pathway. Insulin (100 nM) enhanced ERK1/2 phosphorylation, and the effect on ERK2 phosphorylation was significantly prevented by DPPE $(30 \mu \mathrm{M})$ (Fig. 4C). This suggests that DPPE could inhibit insulin-induced ERK2 activation by facilitating PP2A-mediated dephosphorylation of threonine phosphorylation of ERK2.

DPPE inhibits insulin-stimulated GLUT4 translocation and glucose uptake into cells

Akt is a key factor for GLUT4 translocation to the cell surface. If DPPE inhibits insulininduced Akt1/2 activation, then it should prevent insulin-stimulated GLUT4 translocation to the cell surface. We therefore examined the effect of DPPE on GLUT4 translocation in 3T3L1-GLUT4myc adipocytes. Insulin (100 nM) significantly increased GLUT4 localization on 
Fig. 5. Effect of DPPE and DAPE on insulin-stimulated GLUT4 translocation to the cell surface in 3T3-L1-GLUT4 myc adipocytes. Cells were untreated and treated with insulin (Ins) $(100 \mathrm{nM})$ in the presence and absence of DPPE $(30 \mu \mathrm{M})(\mathrm{A})(\mathrm{B})$ or DAPE $(30 \mu \mathrm{M})(\mathrm{C})$ (D) for $20 \mathrm{~min}$. (A)(C) Western blotting was carried out in samples separated into the cytosolic (C) and plasma membrane fractions (M) from total cell lysates using an anti-cmyc antibody. In the graphs, each column represents the mean $( \pm$ SEM) ratio of
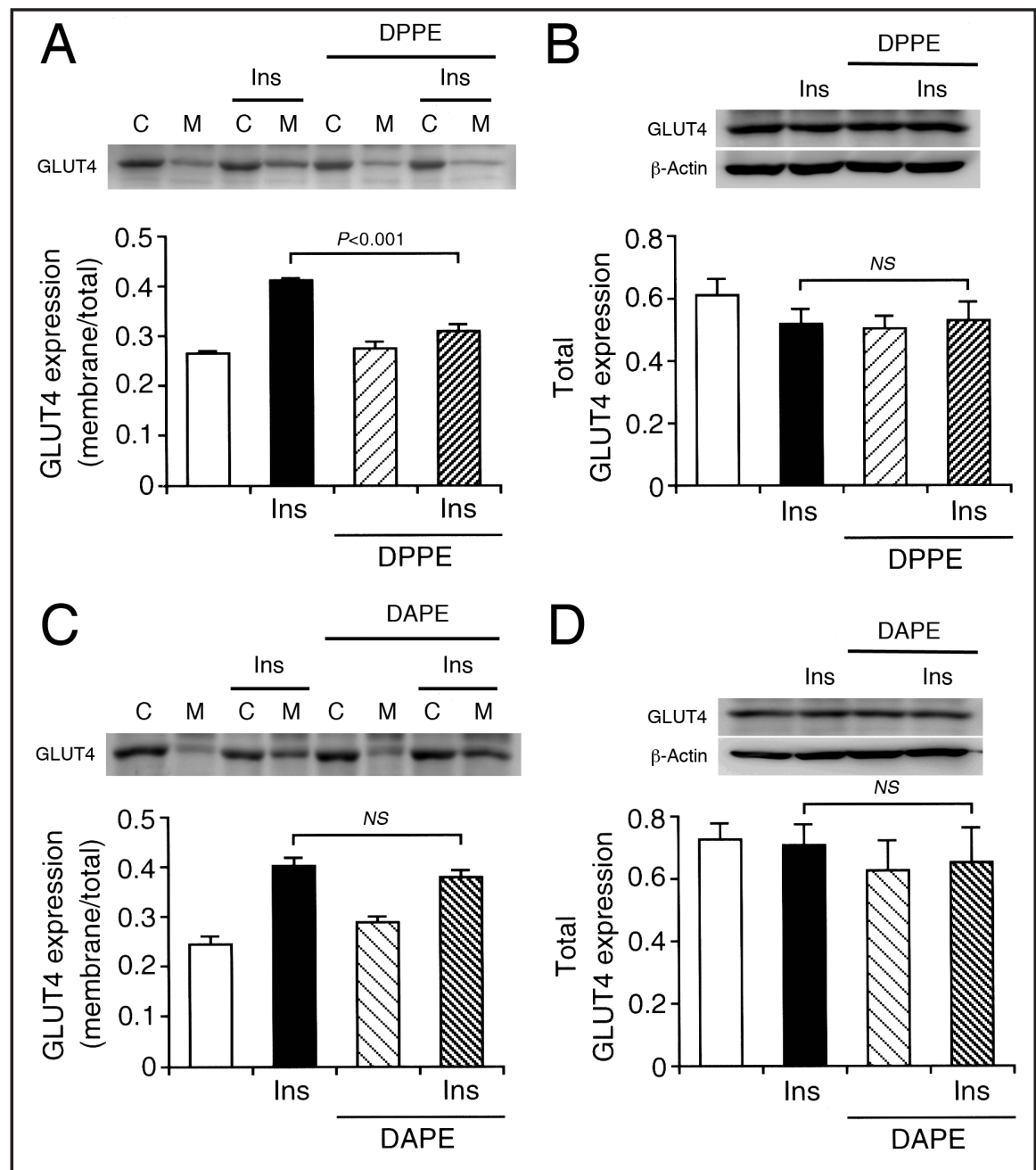

signal intensity for c-myc in the plasma membrane fraction relative to that in the total cell $(\mathrm{n}=4$ independent experiments). (B)(D) Western blotting was carried out in total cell lysates using an anti-c-myc antibody. In the graphs, each column represents the mean $( \pm$ SEM) ratio of signal intensity for c-myc in total cells $(n=4$ independent experiments). $P$ values, ANOVA followed by a Bonferonni correction. $N S$, not significant.

Fig. 6. Effect of DPPE on glucose uptake into 3T3-L1-GLUT4myc adipocytes. Cells were untreated and treated with insulin (Ins)(100 pM) in the presence and absence of DPPE $(30 \mu \mathrm{M})$ in PBS containing glucose $(10 \mathrm{mM})$ for $2 \mathrm{~h}$, and then extracellular glucose was measured by HPLC. In the graph, each column represents the mean ( \pm SEM) glucose uptake (nmol/ $\mu$ g protein $/ \mathrm{min})(\mathrm{n}=4$ independent experiments). $P$ values, Dunnett's rest. NS, not significant.

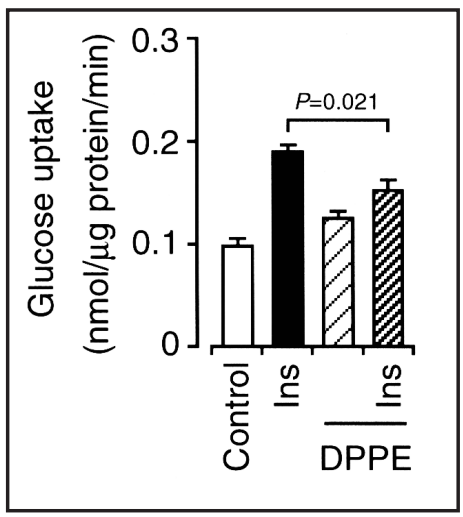

the cell surface (Fig. 5A). DPPE (30 $\mu \mathrm{M})$ abrogated insulin-stimulated increase in the cell surface localization of GLUT4, without affecting total expression of GLUT4 (Fig. 5A,B). In contrast, DAPE $(30 \mu \mathrm{M})$, that has no enhancing effect on PP2A activity, did not affect insulin- 
Fig. 7. A schematic pathway for the action of DPPE on insulin signaling.

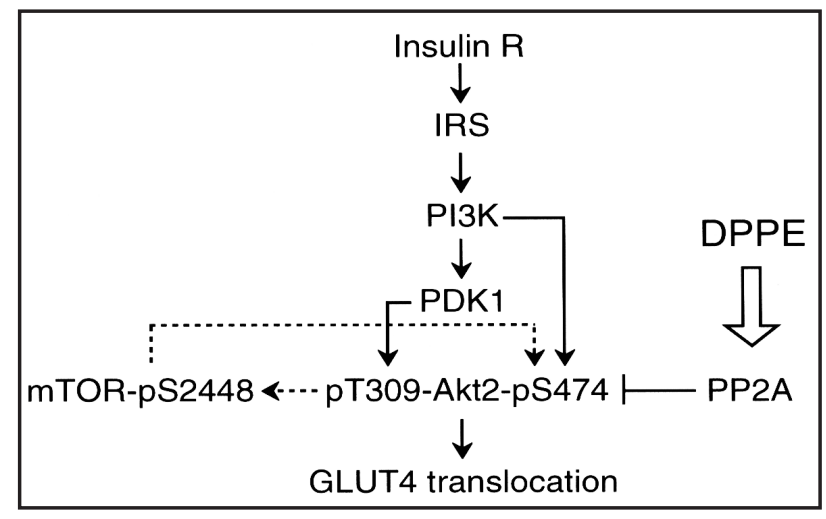

induced increase in the cell surface localization of GLUT4 and total expression of GLUT4 (Fig. $5 \mathrm{C}, \mathrm{D})$.

In the glucose assay, insulin ( $100 \mathrm{pM})$ stimulated glucose uptake into 3T3-L1-GLUT4myc adipocytes, and the effect was significantly prevented by DPPE $(30 \mu \mathrm{M})$ (Fig. 6). Overall, these results indicate that DPPE inhibits insulin-stimulated GLUT4 translocation to the cell surface and glucose uptake into cells.

\section{Discussion}

One of the major finding is that of phosphatidylethanolamines examined here DLPE and DPPE enhanced PP2A and PTP1B activities, with the higher potential for DPPE than for DLPE, while PP1 activity was not affected. DAPE, DLPE, DOPE, and DPPE contain arachidonic acid (20:4), linoleic acid (18:2), oleic acid (18:1), and palmitoleic acid (16:1) both at the $\alpha$ (1) and $\beta$ (2) positions, respectively (Fig. 1). This suggests that the potentiating action of DLPE and DPPE on PP2A and PTP1B activities is independent of the number of carbon atoms of the fatty acid chain or double bonds. Amazingly, we have found that unsaturated FFAs oleic acid, linoleic acid, and linolenic acid (18:3) serve as a strong inhibitor of PTP1B and that saturated FFAs myristic acid (C14:0), palmitic acid (C16:0), stearic acid (C18:0), and behenic acid (C22:0) suppress PP2A and PTP1B activities [12]. How DLPE and DPPE enhances PP2A and PTP1B activities and conversely, how unsaturated and saturated FFAs suppress PP2A and PTP1B activities are presently far from understanding. To address these questions, we are currently attempting further intensive experiments.

Insulin initiates a PI3K/PDK1/Akt signal following tyrosine phosphorylation of its own receptor and IRS-1, to stimulate GLUT4 delivery to the cell surface and glucose uptake into cells. PTP1B inactivates insulin receptor and IRS-1 by dephosphorylating tyrosine phosphorylation of them. In the present study, DPPE reduced insulin-induced tyrosine phosphorylation of insulin receptor and IRS-1, without affecting expression of insulin receptor and IRS-1, in 3T3-L1-GLUT4myc adipocytes, but unexpectedly the inhibitory effect was not significant. This suggests that DPPE could not sufficiently attenuate insulin signaling through PTP1B-mediated tyrosine dephosphorylation of insulin receptor and IRS-1.

DPPE, on the other hand, significantly reduced insulin-induced phosphorylation of Akt1/2 both at Thr308/309 and Ser473/474 in 3T3-L1-GLUT4myc adipocytes. This indicates that DPPE obstructs insulin signaling through PP2A-mediated serine/threonine dephosphorylation and inactivation of Akt1/2. In addition, DPPE significantly reduced insulin-induced phosphorylation of mTOR at Ser2448 in 3T3-L1-GLUT4myc adipocytes. Insulin activates mTOR by phosphorylating at Ser2448 through a pathway along an insulin receptor/IRS-1/PI3K/PDK1/Akt axis [6-9], and activated mTOR phosphorylates Akt1/2 at Ser473/474, which establishes a positive-feedback loop for Akt1/2 activation $[10,11]$. This 
indicates that DPPE-operated mTOR inhibition contributes to further reduction of Akt1/2 activity.

DPPE reduced insulin-induced ERK2 threonine phosphorylation, possibly by facilitating PP2A-mediated dephosphorylation of the phosphorylation. In contrast, insulin-induced ERK1 threonine phosphorylation was not significantly inhibited, although DPPE tended to reduce the phosphorylation. It is presently unknown why DPPE preferentially inhibits ERK2. mTOR functions in two distinct multi-protein complexes. mTOR in complex 1 (mTORC1) contains the regulatory-associated protein of mTOR (raptor) and proline rich Akt substrate $40 \mathrm{kDa}$ (PRAS40) and mTOR in complex 2 (mTORC2) contains rapamycin-insensitive companion of mTOR (rictor) and stress-activated MAP kinase-interacting protein 1 (Sin1) [13]. Recent evidence has pointed to the role of mTORC1 and mTORC2 in the inhibition of c-Jun N-terminal kinase (JNK), a MAP kinase [14]. If DPPE inhibits mTOR, then DPPE should enhance JNK activity. JNK is shown to counteract insulin signaling by phosphorylating IRS1 at Ser307 and neutralizing insulin-induced IRS- 1 activation $[15,16]$. Accordingly, DPPEinduced mTOR inhibition and the ensuing enhancement of JNK activity might also contribute to pathogenesis of insulin resistance.

Akt plays a central role in the regulation of GLUT4 translocation to the cell surface and glucose uptake into cells. In the present study, DPPE inhibited insulin-stimulated GLUT4 translocation to the cell surface in 3T3-L1-GLUT4myc adipocytes, although such effect was not obtained with DAPE, that has no effect on PP2A activity. DPPE also inhibited insulinstimulated glucose uptake into adipocytes. Overall, we drew a conclusion that DPPE inhibits insulin-induced Akt1/2 activation by promoting PP2A-mediated dephosphorylation at Thr308/309 and Ser473/474; DPPE inhibits insulin-induced mTOR activation in association with PP2A-mediated Akt1/2 inhibition, to further reduce Akt1/2 activity; and these inhibitory effects of DPPE on Akt1/2 causes obstruction of the ensuing insulin signaling responsible for GLUT4 translocation to the cell surface and glucose uptake into adipocytes (Fig. 7).

Phosphatidylethanolamine, which is the most abundant lipid on the cytoplasmic layer of cellular membranes, plays significant roles in cellular processes such as membrane fusion [17], cell cycle [18], autophagy [19], apoptosis [20], and cognitive function [21]. Three main biochemical pathways are recognized for production of phosphatidylethanolamine; CDP-ethanolamine Kennedy pathway, mitochondrial phosphatidylserine decarboxylation pathway catalyzed by phosphatidylserine decarboxylase, and acylation of lysophosphatidylethanolamine catalyzed by lysophosphatidylethanolamine acyltransferase. CDP-ethanolamine Kennedy pathway is the only route for de novo synthesis of phosphatidylethanolamine [22]. Phosphorylation of ethanolamine by ethanolamine kinase to produce phosphoethanolamine is followed by the CTP:choline cytidylyltransferase 2 (Pcyt2)-mediated production of CDP-ethanolamine. The final reaction in this pathway is executed by CDP-ethanolamine:1,2-diacylglycerol ethanolaminephosphotransferase to produce phosphatidylethanolamine. The analogous enzymes of the CDP-choline brunch of the Kennedy pathway include choline kinase, Pcyt1, and CDP-choline:1,2-diacylglycerol cholinephosphotransferase. In the liver phosphatidylethanolamine is transformed into phosphatidylcholine catalyzed by phosphatidylethanolamine $N$-methyltransferase. Phosphatidylethanolamine is also produced in mitochondria by phosphatidylserine decarboxylase-catalyzed decarboxylation of phosphatidylserine. Mammals do not synthesize phosphatidylserine de novo; phosphatidylserine is produced by the headgroup exchange from phosphatidylethanolamine catalyzed by phosphatidylserine synthase- 2 or phosphatidylcholine catalyzed by phosphatidylserine synthase- 1 . Moreover, phosphatidylethanolamine is produced by lysophosphatidylethanolamine acyltransferasecatalyzed fatty acid esterification of lysophosphatidylethanolamine. Several avenues of evidence have shown that Pcyt2 knockout mice have metabolic syndrome such as elevated lipogenesis and lipoprotein secretion, hypertriglyceridemia, liver steatosis, obesity, and insulin resistance in the liver [22-24]. Interestingly, the phosphatidylethanolamine content in these mice is not downregulated. Accordingly, insulin resistance found in Pcyt2 knockout mice is likely due to a consequence of deregulation of homeostasis of phosphatidylethanolamine 
biosynthesis rather than to a direct effect of phosphatidylethanolamine. In the present study, DPPE attenuated insulin signaling responsible for GLUT4 translocation to the cell surface and glucose uptake into adipocytes. This suggests that DPPE might be a factor responsible for insulin resistance and progression to type 2 diabetes and that DPPE might worsen metabolic syndrome closely related to insulin resistance and type 2 diabetes. This may interpret that phosphatidylethanolamines exhibit different effects on glucose metabolism, depending upon phosphatidylethanolamine types or cell types, e.g., between hepatocytes and adipocytes. To address this question, we are currently carrying out further experiments.

In conclusion, the results of the present study show that DPPE serves as a potent PP2A enhancer, to inhibit insulin-induced Akt activation by promoting serine/threonine dephosphorylation of Akt and therewith inhibiting mTOR, responsible for GLUT4 translocation to the cell surface and glucose uptake into adipocytes (Fig. 7). This may represent a new action of phosphatidylethanolamines including DPPE on insulin signaling.

\section{Acknowledgements}

We thank to Prof. Ebina (Institute for Enzyme Research, The University of Tokushima, Tokushima, Japan) for providing us with 3T3L1-GLUT4myc fibroblast cell line. We also thank to Prof. Gotoh and Dr. Nagaya (Laboratory of Cell and Gene Therapy, Institute for Advanced Medical Sciences, Hyogo College of Medicine) for experimental advice.

\section{References}

1 Obanda DN, Cefalu WT: Modulation of cellular insulin signaling and PTP1B effects by lipid metabolites in skeletal muscle cells. J Nutr Biochem 2013;24:1529-1537.

-2 Obanda DN, Hernandez A, Ribnicky D, Yu Y, Zhang XH, Wang ZQ, Cefalu WT: Bioactives of Artemisia dracunculus L. mitigate the role of ceramides in attenuating insulin signaling in rat skeletal muscle cells. Diabetes 2012;61:597-605.

3 Feng XT, Wang TZ, Leng J, Chen Y, Liu JB, Liu Y, Wang WJ: Palmitate contributes to insulin resistance through downregulation of the Src-mediated phosphorylation of Akt in C2C12 myotubes. Biosci Biotechnol Biochem 2012;76:1356-1361.

4 Kanno T, Tsuchiya A, Shimizu T, Tanaka A, Nishizaki T: Indomethacin serves as a potential inhibitor of protein phosphatases. Cell Physiol Biochem 2012;30:1014-1022.

-5 Kuo YC, Huang KY, Yang CH, Yang YS, Lee WY, Chiang CW: Regulation of phosphorylation of Thr-308 of Akt, cell proliferation, and survival by the B55 $\alpha$ regulatory subunit targeting of the protein phosphatase $2 \mathrm{~A}$ holoenzyme to Akt. J Biol Chem 2008;283:1882-1892.

6 Bolster DR, Crozier SJ, Kimball SR, Jefferson LS: AMP-activated protein kinase suppresses protein synthesis in rat skeletal muscle through down-regulated mammalian target of rapamycin (mTOR) signaling. J Biol Chem 2002;277:23977-23980.

7 Reynolds TH4th, Bodine SC, Lawrence JCJr: Control of Ser2448 phosphorylation in the mammalian target of rapamycin by insulin and skeletal muscle load. J Biol Chem 2002;277:17657-17662.

-8 Sekulić A, Hudson CC, Homme JL, Yin P, Otterness DM, Karnitz LM, Abraham RT: A direct linkage between the phosphoinositide 3-kinase-AKT signaling pathway and the mammalian target of rapamycin in mitogenstimulated and transformed cells. Cancer Res 2000;60:3504-3513.

-9 Withers DJ, Ouwens DM, Nave BT, van der Zon GC, Alarcon CM, Cardenas ME, Heitman J, Maassen JA, Shepherd PR: Expression, enzyme activity, and subcellular localization of mammalian target of rapamycin in insulin-responsive cells. Biochem Biophys Res Commun 1997;241:704-709.

10 Bayascas JR, Alessi DR: Regulation of Akt/PKB Ser473 phosphorylation. Mol Cell 2005;18:143-145.

11 Gao T, Furnari F, Newton AC: PHLPP: a phosphatase that directly dephosphorylates Akt, promotes apoptosis, and suppresses tumor growth. Mol Cell 2005;18:13-24.

12 Shibata E, Kanno T, Tsuchiya A, Kuribayashi K, Tabata C, Nakano T, Nishizaki T: Free fatty acids inhibit protein tyrosine phosphatase 1B and activate Akt. Cell Physiol Biochem 2013;32:871-879. 
13 Ferrari S, Pearson RB, Siegmann M, Kozma SC, and Thomas G: The immunosuppressant rapamycin induces inactivation of p70s6k through dephosphorylation of a novel set of sites. J Biol Chem 1993;268:1609116094.

14 Martin TD, Dennis MD, Gordon BS, Kimball SR, Jefferson LS: mTORC1 and JNK coordinate phosphorylation of the p70S6K1 autoinhibitory domain in skeletal muscle following functional overloading. Am J Physiol Endocrinol Metab 2014;306:E1397-405.

15 Guo S: Insulin signaling, resistance, and the metabolic syndrome: insights from mouse models into disease mechanisms. J Endocrinol 2014;220:T1-T23.

-16 Taniguchi CM, Emanuelli B, Kahn CR: Critical nodes in signaling pathways: insights into insulin action. Nat Rev Mol Cell Biol 2006;7:85-96.

17 Deeba F, Tahseen HN, Sharad KS, Ahmad N, Akhtar S, Saleemuddin M, Mohammad O: Phospholipid diversity: Correlation with membrane-membrane fusion events. Biochim Biophys Acta 2005;1669:170181.

18 Emoto K, Kobayashi T, Yamaji A, Aizawa H, Yahara I, Inoue K, Umeda M: Redistribution of phosphatidylethanolamine at the cleavage furrow of dividing cells during cytokinesis. Proc Natl Acad Sci USA 1996;93:12867-12872.

19 Ichimura Y, Kirisako T, Takao T, Satomi Y, Shimonishi Y, Ishihara N, Mizushima N, Tanida I, Kominami E, Ohsumi M, Noda T, Ohsumi Y: A ubiquitin-like system mediates protein lipidation. Nature 2000;408:488492.

20 Emoto K, Toyama-Sorimachi N, Karasuyama H, Inoue K, Umeda M: Exposure of phosphatidylethanolamine on the surface of apoptotic cells. Exp Cell Res 1997;232:430-434.

21 Yaguchi T, Nagata T, Nishizaki T: 1,2-dilinoleoyl-sn-glycero-3-phosphoethanolamine ameliorates agerelated spatial memory deterioration by preventing neuronal cell death. Behav Brain Funct 2010;6:52. doi:10.1186/1744-9081-6-52.

-22 Pavlovic Z, Bakovic M: Regulation of phosphatidylethanolamine homeostasis-The critical role of CTP:phosphoethanolamine cytidylyltransferase (Pcyt2). Int J Mol Sci 2013;14:2529-2550.

23 Fullerton MD, Hakimuddin F, Bonen A, Bakovic M: The development of a metabolic disease phenotype in CTP: Phosphoethanolamine cytidylyltransferase-deficient mice. J Biol Chem 2009;284:25704-25713.

24 Singh RK, Fullerton MD, Vine D, Bakovic M: Mechanism of hypertriglyceridemia in CTP: phosphoethanolamine cytidylyltransferase-deficient mice. J Lipid Res 2012;53:1811-1822. 